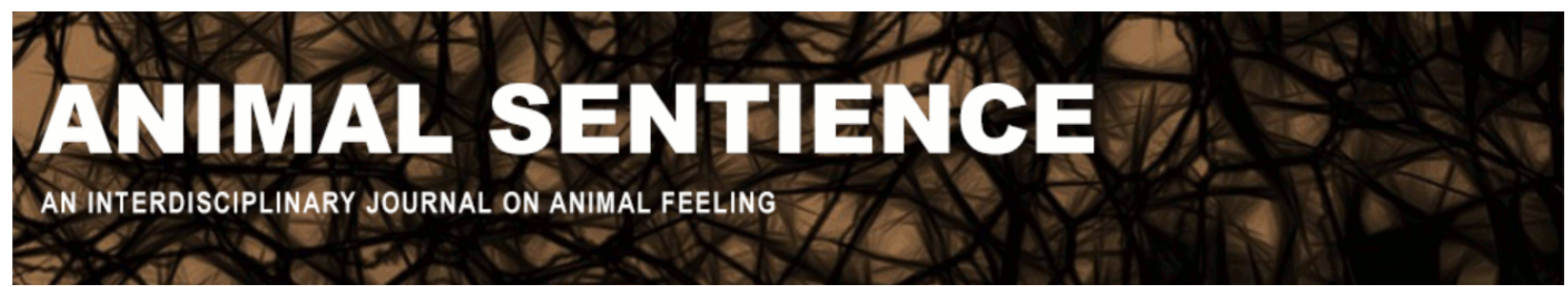

Mallatt, Jon and Feinberg, Todd E. (2016) Insect consciousness: Fine-tuning the hypothesis. Animal Sentience 9(10)

DOI: $10.51291 / 2377-7478.1141$

Date of submission: 2016-08-11

Date of acceptance: 2016-08-17

(c) (†)

This article has appeared in the journal Animal

Sentience, a peer-reviewed journal on animal

cognition and feeling. It has been made open access,

free for all, by WellBeing International and deposited

in the WBI Studies Repository. For more information,

please contact

wbisr-info@wellbeingintl.org.

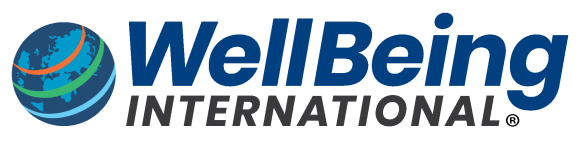

SOLUTIONS FOR PEOPLE, ANIMALS AND ENVIRONMENT 


\title{
Insect consciousness: Fine-tuning the hypothesis
}

\author{
Commentary/Article on Klein \& Barron on Insect Experience
}

\author{
Jon Mallatt \\ School of Biological Sciences \\ Washington State University \\ Todd E. Feinberg \\ Clinical Psychiatry and Neurology \\ Albert Einstein College of Medicine
}

\begin{abstract}
Although we are mostly supportive, we point out the strengths and weaknesses of Klein \& Barron's (2016) hypothesis that insects have the most basic form of consciousness. The strengths are in their application of Bjorn Merker's vertebrate-derived ideas to arthropods, using their deep knowledge of insect brains. The weaknesses involve the controversial aspects of some of Merker's ideas. We describe how the latter can be modified to strengthen the authors' case for insect consciousness.
\end{abstract}

Keywords: affective consciousness, basal ganglia, central complex of insect brain, cerebral cortex, convergent evolution, optic tectum

Jon M. Mallatt is Associate Professor at School of Biological Sciences, Washington State University and University of Washington School of Medicine, His research is on the origin of the major animal groups, especially vertebrates and the origin of the vertebrate jaw. He has worked with Todd Feinberg on the origin and nature of consciousness since 2013.

http://public.wsu.edu/ jimallatt/

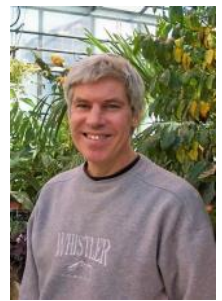

Todd E. Feinberg, Professor of Clinical Psychiatry and Neurology at Albert Einstein College of Medicine, is internationally recognized as a leading authority on how the neurobiology of the brain creates the individual's sense of identity. http://www.toddfeinberg.com

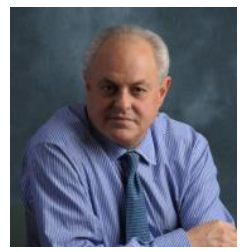

Klein \& Barron (2016) (henceforth K \& B) propose that insects and all the other arthropods have primary consciousness, as do all the vertebrates, judging from some similarities of brain structure and function in these two great clades of animals. We have written a book (Feinberg \& Mallatt, 2016a) with similar ideas, published at almost the same time as K \& B's original presentation of their thesis in PNAS in March/April of this year (Barron \& Klein, 
2016). Our book focused more on vertebrates whereas K \& B focused on insects, so the two works nicely complement each other.

Our first major point of agreement is that we all view consciousness as a natural phenomenon that can be explained by empirical science without invoking any new fundamental forces.

Second, we agree - contrary to the dominant view in consciousness studies today (Koch, Massimini, Boly, \& Tononi, 2016) - that consciousness does not need a mammalian cerebral cortex, but can instead come from subcortical regions of the midbrain and forebrain of vertebrates as well as from comparable brain regions of insects.

Third, we really do agree with K \& B on the likelihood of insect consciousness: starting with vertebrates we listed twelve traits that indicate the presence of consciousness, and then found that insects have all but one of them (see the Tables in Chapter 9 of Feinberg \& Mallatt, 2016a).

We did point out the one way insects failed, in that their tiny brains may be too small for consciousness, because this is sure to be an ongoing point of contention and doubt in the field. However, we concluded that insects are "probably conscious" and we largely treated them as such through the rest of our book. Thus, we are not major doubters, as K \& B seem to imply by saying we are "equivocal on whether insects have the capacity" for consciousness (p. 9).

\title{
A. Strong points of their theory
}

A1. The idea is coherent and interesting. To quote from pages 7 and 8 of $\mathrm{K} \& \mathrm{~B}$ :

\begin{abstract}
"In summary, there are significant parallels between the functional organization of the insect brain and that of the vertebrate midbrain [and basal ganglia as a] behavioral core control system. Both systems have specialized regions for processing the position of the moving animal in space. In both systems, action selection is resolved by combining information on [body] position with information on the environment, the relevance of stimuli in the environment to the animal, and the [inner physiological] state of the animal. . . the channels of information are sufficiently unified such that the system as a whole creates a functional representation of the state of the mobile animal in space as a solution for effective decision making. . . processing of this kind supports the capacity for a subjective experience of the environment."
\end{abstract}

This fits our idea that the brain region for primary consciousness receives and integrates information from the many kinds of complex senses and builds this input into a map-like representation of the world and body, a subjective mental image that is used to guide and direct the behavior of the conscious animal. Our view emphasizes the sensory side of the equation whereas K \& B follow Bjorn Merker $(2005,2007)$ in emphasizing the motor side; but we certainly recognize the strong correlation between consciousness and high mobility in the animal phyla they pointed out. 
Our original idea was that conscious animals use their complex senses of vision, hearing, smell, touch, etc. to model the world when they are immobile and still as well as when they are moving, meaning mobility cannot be the whole story. But mobility is important, and we like the Merker-inspired statement that consciousness evolved to "create a neural simulation of the state of the mobile animal in space" (Barron \& Klein, 2016). It adds a helpful new dimension to our thinking. In fact, Merker, Barron, and Klein inspired us to add the following to our list of traits for judging whether an animal clade has consciousness: "Demonstrated ability to select actions directed toward a targeted goal in space (even without a continuous stimulus to follow)." This ability includes elements of planning, of feeling an attraction, and motivation, as well as mentally mapping the world. This case is best presented on pages 10-11 of K \& B's target article.

A2. Convincing comparison of insect and vertebrate brain regions for consciousness. As $\mathrm{K} \& \mathrm{~B}$ have shown, the central complex of the insect brain (CX) receives and processes mapped information from almost all the senses and uses this sensory information for spatial orientation, as does the superior colliculus/optic tectum of the vertebrate brain. Thus $\mathrm{K} \& \mathrm{~B}$ logically equate these as sites of mental-image formation. Besides this " $\mathrm{CX}=$ tectum" equation, K \& B also showed that the CX and nearby "protocerebrum" are where different types of sensory input compete to decide which motor program to perform. Such decision making for action selection is likewise performed by the basal ganglia of the vertebrate brain. Thus it is logical to equate the CX and "protocerebrum" with the basal ganglia, for consciousness-driven action. They also document the involvement of CX and "protocerebrum" in rewards and motivations for goal-directed behaviors, another similarity to the vertebrate basal ganglia. Their detailed knowledge of arthropod brains allowed K \& B to identify specific brain areas and use them as evidence for insect consciousness. This is an advance over what we accomplished in our book.

To strengthen K \& B's case even more, we will point out several more similarities between insects and vertebrates - in olfaction, memory, and the affective feelings of like and dislike. The olfactory similarities will be considered first.

In insects the olfactory pathway differs from the other sensory pathways in sending little or no input to the CX on its way to the higher brain (mushroom bodies: Pfeiffer \& Homberg, 2014). Similarly, in fish and amphibians olfaction is the only sense whose main projection is not to the optic tectum (the CX-equivalent) but to the higher brain (the cerebral pallium, which is the homologue of the cortex of mammals: Miyasaka et al., 2014; Mori, Manabe, Narikiyo, \& Onisawa, 2013; Wullimann \& Vernier, 2009).

Turning to the similarities in memory, much olfactory information goes directly to the brain's main regions for memory and learning in both insects and vertebrates, to the mushroom bodies and the hippocampus, respectively (Brodal, 2010; Galizia, 2014). Such a strong association between the smell and memory circuits should be universal among conscious animals because smell is the only sense whose stimuli linger, meaning the scent must be remembered in order for a long-gone source to be identified (pp. 84 and 115, Feinberg \& Mallatt, 2016a). 
Regarding affective consciousness, we searched the literature and found behavioral evidence that insects experience basic likes and dislikes, as do all the vertebrates (Chapters 8 and 9, Feinberg \& Mallatt, 2016a). More specifically, insects meet five criteria, most of which are based on an expert capacity to learn operantly to approach rewards and avoid punishments. Thus, the behavioral evidence buttresses $\mathrm{K}$ \& B's claim for insect consciousness.

$\mathrm{K} \& \mathrm{~B}$ recognize that arousal and selective attention are important for consciousness; they list some brain regions for these processes in insects. They have not compared these to vertebrate regions, however, so we will offer a few preliminary comparisons. Arousal determines the level of consciousness, and in insects, dopaminergic neurons in the CX are involved in it (Liu et al., 2012; Pfeiffer \& Homberg, 2014). In vertebrates, on the other hand, the reticular formation in the brainstem is involved, as is the basal forebrain; the relevant neurons produce acetylcholine and norepinephrine (Fuller et al., 2011). Other than having medial locations in the brain, these arousal-associated structures of insects and vertebrates show no obvious similarities. Attention is a part of, or closely related to, consciousness because sensations are most likely to enter consciousness when attended to. In insects the attention circuits are in the "protocerebrum" (p. 4904, Barron \& Klein, 2016), with visual attention also involving early processing in the optic lobes (Paulk et al., 2004). In fish and amphibians, attention involves the isthmus nuclei in the brainstem interacting with the nearby optic tectum (Dutta \& Gutfreund, 2014; p. 110, Feinberg \& Mallatt, 2016a; Gruberg et al., 2006). These regions may be comparable in insects and vertebrates, although the vertebrate tectum is farther along the visual pathway than are insect optic lobes. More studies are needed.

A3. Primary consciousness. K \& B nicely explained the concept of primary consciousness as the minimal level of subjective experience or "basic awareness of the world without further reflection on that awareness." Inspired by Morin (2006) and Merker (2007), K \& B state it especially well, making it clear that insects do not monitor their own cognitive processes nor are they self-aware. K \& B were wise to say that insects probably have nothing comparable to the cerebral cortex of mammals. This clarification should prevent a lot of confusion in future critiques of their work: they do not claim that insects have human higher consciousness. We had missed the "without further reflection" part in our own definition of primary consciousness, and appreciate that K \& B emphasize it.

A4. Lower limit on consciousness. Those of us who believe that animals other than humans are conscious face the problem of finding the lower limit. Are rats conscious? Reptiles? Fish? Worms? Amoebas? Bacteria? K \& B help to set this limit with their argument that nematode roundworms, representing the primitive state of (bilaterian) animals, cannot be conscious due to limitations of sensory detection and sensory processing. With just simple photo-, chemo-, and mechano-receptors, nematodes "have no spatial senses. They have no capacity to locate themselves in their environment or to detect the relative position of objects around them" (p. 10). They do not simulate the world around them into a big picture, so they cannot form goals or anticipatory plans of how to act in the world. They use an 
undirected food-foraging strategy called area-restricted search (Hills, 2006) and cannot perform directed motions except in response to immediate stimuli.

Most of the other invertebrate groups also lack elaborate eyes, olfactory organs, ears, etc. so they also fall in this non-conscious category. We came to the same conclusion, but adding $\mathrm{K}$ \& B's idea that animals need to "model the world in order to plan targeted behaviors" adds a strong new dimension to the explanation.

A5. Tectal interactions with the basal ganglia. We did not know that the superior colliculus/optic tectum extensively communicates back and forth with the basal ganglia. We appreciate K \& B pointing this out because it means that the tectum, with its world map based on multisensory inputs, can influence which of the stored motor programs will be executed. It explains how primary consciousness can lead to complex motor sequences and behaviors. $\mathrm{K} \& \mathrm{~B}$ cite the references on mammals, but the tectal-basal ganglia interconnections seem to characterize all vertebrates because they are also documented in fish and amphibians (de Arriba \& Pombal, 2007; Marin, González, \& Smeets, 1997; Wilczynski \& Northcutt, 1983).

\section{B. Constructive criticisms}

Just as we ourselves have trouble mastering the intricate brains of insects, K \& B struggle with the complex structure and terminology of vertebrate brains. Perhaps this is because Merker (2007), their main source, is not a simple read, with its added flourishes, new synonyms, and neologisms along with its many original ideas. Adopting Merker's "behavorial core control system" (BCCS) as the site of primary consciousness and actions, K \& B consider this BCCS to comprise much of the midbrain and diencephalon, plus the basal ganglia (see their Figure 1B). However, they often misleadingly call it all the "midbrain," even though it is much more than that. The real midbrain is shown in yellow in our Figure 1; it contains only a few of the BCCS elements. At other times, K \& B call it "midbrain and basal ganglia," which is better, but that name omits the diencephalon. Thus, as their BCCS they actually mean "basal ganglia plus midbrain+diencephalon" (with the latter shown as a gray S-band in Figure 3 of Merker, 2007). The imprecise naming forces the reader to do a lot of mental translation to understand the structures meant. Perhaps K \& B can straighten out the wording when presenting their ideas in the future. As an aid, our book includes a primer on vertebrate brain anatomy and terminology (Feinberg \& Mallatt, 2016a), so it can help scientists to make more precise comparisons between vertebrate and insect brains. To this end, our book avoids the traditional overemphasis on mammalian brains, centering on the more relevant brains of the basally evolved fishes and amphibians. 


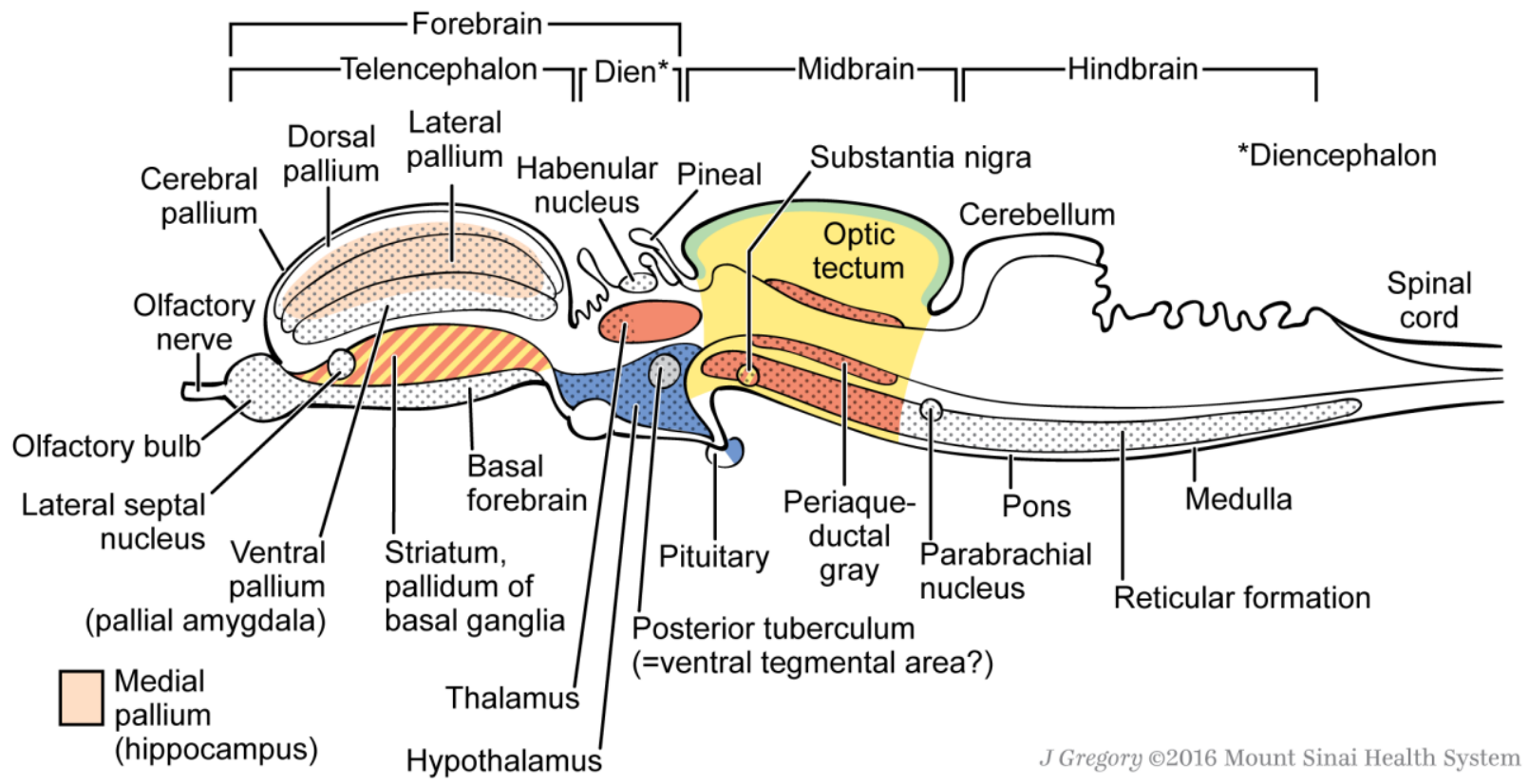

Figure 1: Generalized vertebrate brain. We argue that $K \& B$ have not included enough brain areas in their core region for primary consciousness and behavioral control. They often call it "midbrain" (actually just the subpart shown in yellow) but they mean "midbrain + diencephalon + basal ganglia" (all regions shown in the red, blue, yellow and green). The regions for affective consciousness and its directed actions (stippling) must be included as well. Thanks to Jill K. Gregory for the art.

It seems to us that $\mathrm{K} \& \mathrm{~B}$, like Merker, put too much emphasis on the unity and integration of consciousness, as if it were one thing generated by a spatially confined "core" brain region. We on the other hand see consciousness as diverse, with semi-distinct exteroceptive, interoceptive, and affective aspects originating from different brain regions that are widely distributed (although interconnected and intercommunicating).

We can anticipate a rebuttal to this criticism. That is, $\mathrm{K} \& \mathrm{~B}$ do say that primary consciousness has several aspects, as adopted from Merker (see Figure 1A in K \& B, 2016). The first of these aspects is "environmental state and structure," which is our exteroceptive consciousness of mapped mental simulations of the outside world, as built from the distance senses of seeing, hearing, smelling, etc. Their second aspect is "state of self," which is the sensory awareness of internal physiological processes that we called interoceptive consciousness. Their third aspect is consciously sensing self-movements through proprioception and the sense of balance. And their fourth is the contribution of memories to the conscious experience. We have not explicitly put these third and fourth aspects into our scheme but we recognize their importance in primary consciousness (pp. 114 and 210, Feinberg \& Mallatt, 2016a), so we heartily support including them.

Whereas both K \& B and ourselves subdivided consciousness, K \& B could have done more to analyze whether the different aspects relate to different regions in the insect brain. Their analysis misses, or at least underemphasizes, the important aspect of affective consciousness of dislikes and likes. In vertebrates, affective consciousness mostly involves 
brain regions different from those of the exteroceptive consciousness of the external world (Figure 1; also see Chapters 6-8, Feinberg \& Mallatt, 2016a).

Of course, all aspects of consciousness become united in the end before they reach one's experience - we realize that. Our points are just that in the future (1) it will help to examine the insect brain for the specific subparts associated with interoceptive, exteroceptive, and affective consciousness; and (2) the affective component should be given more emphasis, to allow better comparison with vertebrate brains.

\section{Merker under the microscope}

Some of the strengths of K \& B's thesis, as well as some of its weaknesses, can be traced to its foundation in Merker's work. Only by closely analyzing Merker can one find how to make the valuable hypothesis of insect consciousness even better.

C1. Strengths. Merker $(2005,2007)$ was a trailblazer, formulating a theory of how consciousness evolved in the tectum of the earliest vertebrates years before we and other workers published this conclusion (Feinberg, 2012; Feinberg \& Mallatt, 2013; Packard \& Delafield-Butt, 2014). Besides coming up with the idea that consciousness served to simulate the spatial environment as the fishlike pre-vertebrates swam through it, he also saw that sharp vision was the first of the vertebrate distance senses to evolve (pp. 69-70, Merker, 2007), a feat for which we did not give him enough credit in past works (Feinberg \& Mallatt, 2013; 2016a,b).

Merker's "reafference" theory of the origin of primary consciousness is quite good; K \& B also present it well. The theory says that for swimming pre-vertebrates to see an object as fixed and steady while their swimming movements swivelled their heads, they evolved tectum-based reflexes for compensatory eye movements so the gaze stayed on one point. Next, as other distance senses evolved for monitoring sounds, vibrations and electrical fields in the water, these sensory inputs also reached the tectum, as fully aligned with the mapped visual input. The integrated multisensory mapping evolved not only to enrich the information content of the spatial sensory image but also because, "For active animals with well-developed spatial senses, it is computationally more effective to resolve the reafference problem once in a unified sensory model than to resolve it in a dispersed and peripheral way for each sense independently" (p. 3, K \& B). Whereas Merker (2007, p. 69) documented reafference in mammals, Barron \& Klein (2016, p. 5) documented it in the visual system of insects (also see Kim, Fitzgerald, \& Maimon, 2015).

Another strength of Merker is pointing out that children born without a cerebral cortex (a condition called hydranencephaly) still show strong signs of emotional expression. This is good evidence that affective consciousness arises subcortically in "limbic" structures in humans (Aleman \& Merker, 2014; Nieuwenhuys, 1996). But consciousness has different subtypes, so it does not follow that human exteroceptive consciousness also arises subcortically like this. 


\section{C2. Weaknesses.}

The claim that mammalian primary consciousness is tectal not cortical. Only mammals have an elaborated cerebral cortex, or neocortex, of the pallial forebrain. By contrast, other vertebrates have either a differently elaborated pallium (e.g., birds, galeomorph sharks, hagfish), or a simpler, less elaborated pallium (amphibians and most fishes). Most investigators have for centuries located consciousness in the cerebral cortex. To this day, the dominant paradigm in consciousness studies is that primary consciousness of mapped mental images in mammals comes from the cerebral cortex or from interactions between the cortex and the thalamus, not from the superior colliculus/tectum as Merker claims. We agree with the dominant paradigm for mammals because so many of the neural correlates of mammalian exteroceptive consciousness are in this corticothalamic system (Koch, Massimini, Boly, \& Tononi, 2016). Medical neuroimaging and brain-lesion studies strongly support cortical consciousness when the results are interpreted in the most direct and straightforward way: damage to the cortex leads to loss of some sensory consciousness (Boly et al., 2013; Feinberg, 2009). Destruction of the visual, occipital, cortex causes blindness in primates. In his argument for tectal instead of cortical consciousness, Merker (2007) said that these loss phenomena are more complex than they appear, that the cortical damage actually inhibits the conscious role of the superior colliculus, etc. However, his interpretation is less parsimonious and therefore it requires extraordinary counterevidence to be believed. The indirect counterevidence that Merker provided - on the "Sprague effect" (p. 67) and on the cortex projecting to the superior colliculus (p. 76) - does not seem definitive enough to topple the dominant view of cortical consciousness in mammals.

K \& B recognize that most neurobiologists reject Merker's unorthodox idea of tectal consciousness in mammals, but they defend it with the argument that there is a distinction between the contents of consciousness (from cerebral cortex) and the capacity for it (in superior colliculus). Yet this seems confusing. How could consciousness exist if it had no contents?

Our own solution to the problem raised by the convincing evidence for cortical consciousness is that the consciousness shifted from the tectum to the enlarging cerebral cortex when mammals evolved from their reptile-like ancestors. A more precise way to say this is that all non-olfactory exteroceptive consciousness shifted, some parts of interoceptive consciousness shifted, and cognitive control over affective consciousness shifted from subcortical to cortical regions. We definitely agree, however, with Merker's claim for tectal consciousness in the basally evolved vertebrate clades of fish and amphibians. In fact, much of our work has involved building the case for tectal consciousness in basal vertebrates. Moreover, to reiterate, we do accept Merker's evidence from the hydranencephalic children that primary affective consciousness is not cortical but subcortical in every vertebrate - which we can say because most of the affect-associated brain structures are highly conserved across Vertebrata.

In summary, here is how our conclusions compare to those of Merker, Barron, and Klein. Merker said the tectum is for exteroceptive consciousness of spatial images in mammals and all other vertebrates; he did not say much about affective or interoceptive 
consciousness. We say the cerebral cortex is for exteroceptive consciousness in mammals, but the tectum has this role in fish and amphibians, while affective consciousness arises from subcortical structures in all vertebrates (shaded in Figure 1).

Too mammal centered. As another possible weakness, Merker got almost all his evidence for midbrain+diencephalic consciousness, and for its origin in the first vertebrates, from these brain structures in humans and other mammals. This is ironic in light of the evidence just mentioned that mammals do not have tectal consciousness. Furthermore, the mammalian tectum has some potentially odd features: it is secondarily reduced in size, and its extensive back-and-forth communication with the cerebral cortex is unique among vertebrates ( $p$. 122, Feinberg \& Mallatt, 2016a). The brains of the fish and amphibians that may actually have tectal consciousness should have been considered more (Nieuwenhuys, ten Donkelaar, \& Nicholson, 1998), even though they are less studied than mammalian brains. To his credit, Merker built his ideas on the most conserved brain regions of mammals, the regions that all vertebrates possess, and this proved fruitful. In the end, luck was involved because the mammalian superior colliculus proves not to be so odd and it performs many tectal functions that are universal among vertebrates. It has some value as a model after all. However, quite a bit is now known about the tecta of fish and amphibians, with the tecta of lampreys and zebrafish being especially informative (Bianco \& Engert, 2015; Kardamakis, Saitoh, \& Grillner, 2015; Northmore, 2011; Saidel, 2009). For more on the optic tecta of all vertebrates see our book (pp. 108-111, Feinberg \& Mallatt, 2016a,).

Missed the basal ganglia for action control. Merker did not include enough about the basal ganglia in his "behavioral core control system" for primary consciousness and action control. This is not meant to fault him because he was writing before the central role of the basal ganglia in action selection and behavioral motivation was widely appreciated. Instead, he assigned these functions to the periaqueductal gray of the midbrain and the hypothalamus. Actually, he did bring in a small part of the "action-related" basal ganglia, namely, the midbrain's substantial nigra (p. 71, Merker, 2007), but this was briefly stated, not mentioned further, and is not shown in the summary figures of his midbrain+diencephalic core.

$\mathrm{K} \& \mathrm{~B}$ recognized the absolute necessity of adding all the basal ganglia to Merker's actioncentered hypothesis, and they did it well. However, most of the basal ganglia lie up in the telecephalon, which expands the behavioral core uncomfortably, far beyond the tight midbrain+diencephalic zone envisioned by Merker.

Merker's brain system was much too small. The newer literature has identified many more parts of the vertebrate brain for controlling actions, and many more brain regions for affective consciousness (Figure 1). More specifically, O'Connell \& Hoffman (2011) identified a decision-making network in the vertebrate brain. This has two parts. The first part is a mesolimbic reward system for evaluating the salience of external stimuli. This system brings in telencephalic structures such as the ventral pallidum (pleasure hot spot), a part of the amygdala ("fear almond"), plus the dopaminergic VTA (ventral tegmental area of the midbrain). The second part is a social-behavioral network that regulates adaptive behavioral actions and includes more parts of the hypothalamus, the midbrain's periaqueductal gray, 
plus the lateral septal nucleus of the forebrain. Again, all this is much more than Merker's small region at the top of the brainstem; it even includes parts of the cerebral cortex because in mammals the hippocampus and some of the amygdala technically belong to the cortex.

The challenge this expansion offers to $\mathrm{K} \& \mathrm{~B}$ is that it presents many more vertebrate brain regions whose insect analogues must be found in order to complete their hypothesis of primary consciousness and action selection in insects. We are confident that such matches can be made, but it makes the exercise more difficult, especially for matching the affective and memory regions in the brains of the two clades. As K \& B recognized, there will also be differences; perfect one-to-one correspondence between insect and vertebrate structures is not to be expected. For example, do the memory-related amygdala and hippocampus of vertebrates resemble the memory-related mushroom bodies of insects, or do they resemble parts of the central complex, or the "protocerebrum" (Barron et al., 2015; Krashes et al., 2009; Plath \& Barron, 2014)?

\section{Homology or convergence?}

In Section 4 of their paper K \& B parallel us in showing that the conscious brain regions existed in both the first arthropods and the first vertebrates of about 520 million years ago. Then they asked whether this means that the common ancestor of these two clades was conscious. They are non-committal but lean toward a qualified "yes."

"It is presently unclear whether the insect and vertebrate behavioral core control systems evolved independently. Strausfeld and Hirth (2013) have argued for a deep homology between the insect CX and associated structures and vertebrate basal ganglia. If this interpretation is correct, it would imply that a form of behavioral core control system may even predate the divergence of these groups." (p. 8)

We take the "no" view. Arthropods and vertebrates are only distantly related; they are phylogenetically separated by many clades of animals with simpler nervous systems. This is shown in Figure 2. It indicates that conscious brains arose independently in the prearthropods and pre-vertebrates with the similarities explained by convergent evolution. We have also examined the large-brained and behaviorally sophisticated coleoid cephalopods such as octopuses and squids, and concluded that these molluscs are likely to be conscious. They are not closely related to arthropods or vertebrates, indicating that consciousness evolved independently a third time. 


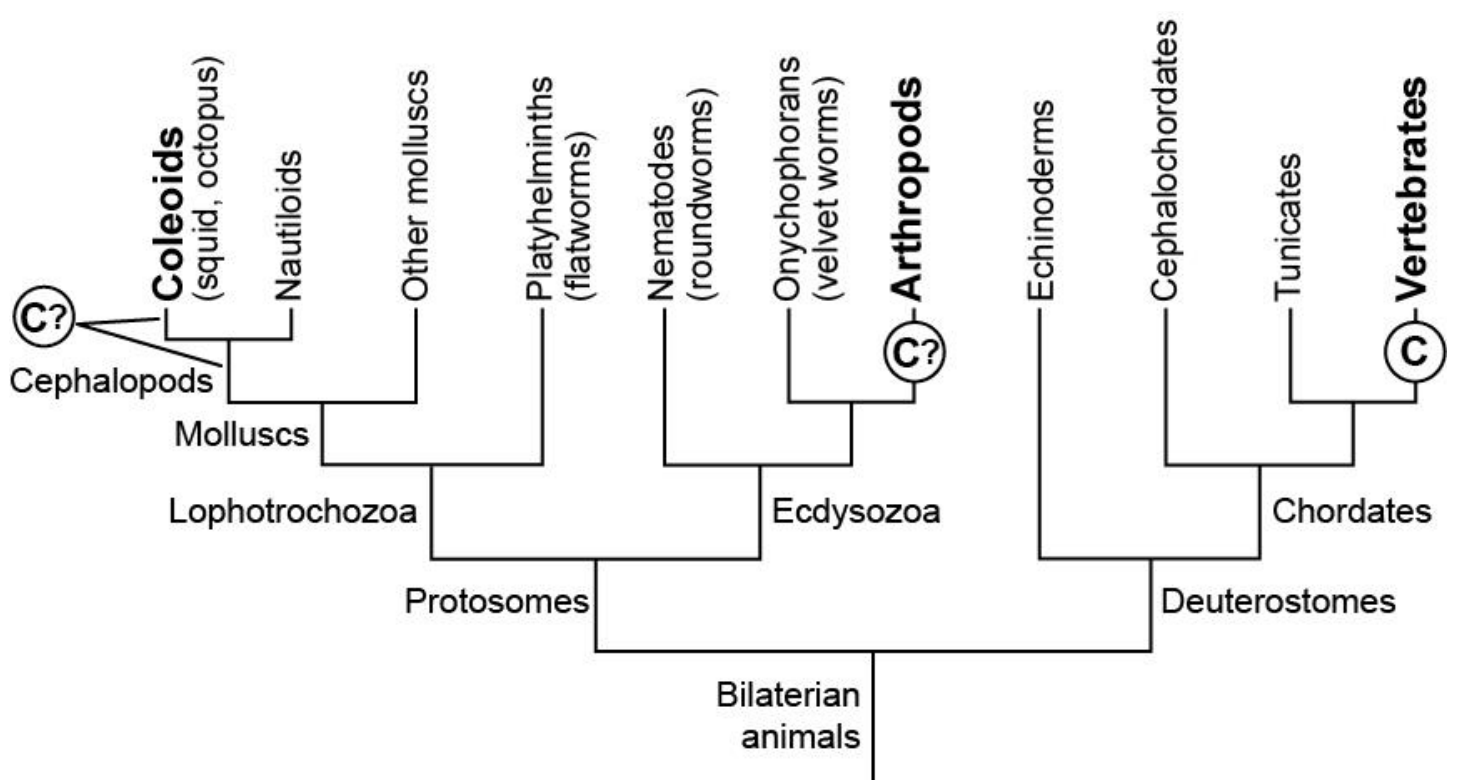

Figure 2: Consciousness (C) evolved independently in vertebrates and arthropods, as shown by the distant relation of these two clades (Dunn, Giribet, Edgecombe, \& Hejnol, 2014). Cephalopods evolved consciousness independently again.

\section{E. Fine-tuning the hypothesis}

Here are our recommendations for adjusting the hypothesis of insect consciousness, fixing some of its rough parts and telling how it may be used and expanded in future inquiry.

Keep the good foundational idea: increasing mobility aided the evolution of primary consciousness in arthropods and vertebrates because consciousness simulates the world so that these animals can accurately navigate and explore their environment. Consciousness directs motile behaviors so the conscious and action-control networks have always been physically near each other in the brain.

The proposed correspondence of insect $\mathrm{CX}$ with vertebrate tectum as the site of primary consciousness of sensory images rings true. However, on the vertebrate side it only applies to the ancestral condition shown in fish and amphibians, given the likelihood that in mammals the cerebral cortex is for this type of consciousness. Thus, future comparisons should use fish data wherever possible.

The proposed correspondence of insect CX and "protocerebrum" with vertebrate basal ganglia as the site of action selection seems very good. Features of the basal ganglia are highly conserved across all vertebrates (Grillner, Robertson, \& Stephenson-Jones, 2013), meaning the literature's heavy focus on mammals will not confuse this comparison.

Separate out affective consciousness and seek more of the affective brain sites in insects. The same could be done for interoceptive consciousness, sensing of the gut and internalbody states. Affects are often associated with distinctive neuromodulators and neurotransmitters, making it easier to identify corresponding sites across animal taxa. 
Locate the particular regions associated with positive versus negative affects in the insect brain.

Realize that even if further study of insect brains finds more similarities with vertebrates, the concept of a unified "behavioral core control system" might fall apart. In the vertebrates this BCCS is much more widespread than originally proposed, with many more brain regions. If this also holds for insects, then it may prove impossible to call anything so complex a unit.

\section{References}

Aleman, B., \& Merker, B. (2014). Consciousness without cortex: A hydranencephaly family survey. Acta Paediatrica, 103, 1057-1065.

Barron, A. B., \& Klein, C. (2016). What insects can tell us about the origins of consciousness. Proceedings of the National Academy of Science of the United States of America, 113(18), 4900-4908.

Barron, A. B., Gurney, K. N., Meah, L. F. S., Vasilaki, E., \& Marshall, J. A. R. (2015). Decisionmaking and action selection in insects: Inspiration from vertebrate-based theories. Frontiers in Behavioral Neuroscience, 9, 216. doi: 10.3389/fnbeh.2015.00216

Bianco, I. H., \& Engert, F. (2015). Visuomotor transformations underlying hunting behavior in zebrafish. Current Biology, 25(7), 831-846.

Boly, M., Seth, A. K., Wilke, M., Ingmundson, P., Baars, B., Laureys, S., \& Tsuchiya, N. (2013). Consciousness in humans and non-human animals: Recent advances and future directions. Frontiers in Psychology, 4.

Brodal, P. (2010). The central nervous system. Structure and function (4th ed.). New York, NY: Oxford University Press.

Chalmers, D. J. (1995). Facing up to the problem of consciousness. Journal of Consciousness Studies, 2, 200-219.

de Arriba Manuel, M. D. C., \& Pombal, A. (2007). Afferent connections of the optic tectum in lampreys: An experimental study. Brain, Behavior and Evolution, 69, 37-68.

Dunn, C. W., Giribet, G., Edgecombe, G. D., \& Hejnol, A. (2014). Animal phylogeny and its evolutionary implications. Annual Review of Ecology, Evolution, and Systematics, 45, 371395.

Dutta, A., \& Gutfreund, Y. (2014). Saliency mapping in the optic tectum and its relationship to habituation. Frontiers in Integrative Neuroscience, 8. 
Feinberg, T. E. (2009). From axons to identity: Neurological explorations of the nature of the self. New York, NY: W.W. Norton.

Feinberg, T. E. (2012). Neuroontology, neurobiological naturalism, and consciousness: a challenge to scientific reduction and a solution. Physics of Life Reviews, 9(1), 13-34.

Feinberg, T. E., \& Mallatt, J. (2013). The evolutionary and genetic origins of consciousness in the Cambrian Period over 500 million years ago. Frontiers in Psychology, 4.

Feinberg T. E., \& Mallatt, J. (2016a). The ancient origins of consciousness. How the brain created experience. Cambridge, MA: MIT Press.

Feinberg, T. E., \& Mallatt, J. (2016b). The nature of primary consciousness. A new synthesis. Consciousness and Cognition, 43, 113-127.

Fuller, P., Sherman, D., Pedersen, N. P., Saper, C. B., \& Lu, J. (2011). Reassessment of the structural basis of the ascending arousal system. Journal of Comparative Neurology, 519(5), 933-956.

Galizia, C. G. (2014). Olfactory coding in the insect brain: Data and conjectures. European Journal of Neuroscience, 39, 1784-1795.

Grillner, S., Robertson, B., \& Stephenson-Jones, M. (2013). The evolutionary origin of the vertebrate basal ganglia and its role in action selection. The Journal of Physiology, 591(22), 5425-5431.

Gruberg, E., Dudkin, E., Wang, Y., Marín, G., Salas, C., Sentis, E., Letelier, J., Mpodozis, J., Malpeli, J., Cui, H., Ma, R., Northmore, D., \& Udin, S. (2006). Influencing and interpreting visual input: The role of a visual feedback system. The Journal of Neuroscience, 26(41), 10368-10371.

Hills, T. T. (2006). Animal foraging and the evolution of goal-directed cognition. Cognitive Science, 30(1), 3-41.

Kardamakis, A. A., Saitoh, K., \& Grillner, S. (2015). Tectal microcircuit generating visual selection commands on gaze-controlling neurons. Proceedings of the National Academy of Sciences, 112(15), E1956-E1965.

Kim, A. J., Fitzgerald, J. K., \& Maimon, G. (2015). Cellular evidence for efference copy in Drosophila visuomotor processing. Nature Neuroscience, 18(9), 1247-1255.

Klein, C., \& Barron A. B. (2016). Insects have the capacity for subjective experience. Animal Sentience 2016.100.

Koch, C., Massimini, M., Boly, M., \& Tononi, G. (2016). Neural correlates of consciousness: progress and problems. Nature Reviews Neuroscience, 17(5), 307-321. 
Krashes, M. J., DasGupta, S., Vreede, A., White, B., Armstrong, J. D., \& Waddell, S. (2009). A neural circuit mechanism integrating motivational state with memory expression in Drosophila. Cell, 139, 416-427.

Lee, S. H., \& Dan, Y. (2012). Neuromodulation of brain states. Neuron, 76(1), 209-222.

Levine, J. (1983). Materialism and qualia: The explanatory gap. Pacific Philosophical Quarterly, 64(4), 354-361.

Liu, Q., Liu, S., Kodama, L., Driscoll, M. R., \& Wu, M. N. (2012). Two dopaminergic neurons signal to the dorsal fan-shaped body to promote wakefulness in Drosophila. Current Biology, 22(22), R949-R951.

Marin, O., González, A., \& Smeets, W. J. (1997). Anatomical substrate of amphibian basal ganglia involvement in visuomotor behaviour. European Journal of Neuroscience, 9(10), 2100-2109.

Merker, B. (2005). The liabilities of mobility: A selection pressure for the transition to consciousness in animal evolution. Consciousness and Cognition, 14, 89-114.

Merker, B. (2007). Consciousness without a cerebral cortex: A challenge for neuroscience and medicine. Behavioral and Brain Sciences, 30, 63-81. doi: 10.1017/S0140525X07000891

Miyasaka, N., Arganda-Carreras, I., Wakisaka, N., Masuda, M., Sümbül, U., Seung, H. S., \& Yoshihara, Y. (2014). Olfactory projectome in the zebrafish forebrain revealed by genetic single-neuron labelling. Nature Communications, 5, 3639.

Mori, K., Manabe, H., Narikiyo, K., \& Onisawa, N. (2013). Olfactory consciousness and gamma oscillation couplings across the olfactory bulb, olfactory cortex, and orbitofrontal cortex. Frontiers in Psychology, 4.

Morin, A. (2006). Levels of consciousness and self-awareness: A comparison and integration of various neurocognitive views. Consciousness and Cognition, 15, 358-371.

Nagel, T. (1974). What is it like to be a bat?. The Philosophical Review, 435-450.

Nieuwenhuys, R. (1996). The greater limbic system, the emotional motor system and the brain. Progress in Brain Research, 107, 551-580.

Nieuwenhuys, R., ten Donkelaar, H. J., \& Nicholson, C. (Eds.). (1998). The central nervous system of vertebrates. Heidelberg: Springer Berlin.

Northmore, D. (2011). The optic tectum. In A. Farrell (Ed.), The encyclopedia of fish physiology: From genome to environment (pp. 131-142). San Diego, CA: Academic Press. 
O'Connell, L. A., \& Hofmann, H. A. (2011). The vertebrate mesolimbic reward system and social behavior network: a comparative synthesis. Journal of Comparative Neurology, 519(18), 3599-3639.

Packard, A., \& Delafield-Butt, J. T. (2014). Feelings as agents of selection: Putting Charles Darwin back into (extended neo-) Darwinism. Biological Journal of the Linnean Society, 112(2), 332-353.

Paulk, A. C., Stacey, J. A., Pearson, T. W. J., Taylor, G. J., Moore, R. J. D., Srinivasan, M. V., \& van Swinderen, B. (2014). Selective attention in the honeybee optic lobes precedes behavioral choices. Proceedings of the National Academy of Science of the United States of America, 111, 5006-5011.

Pfeiffer, K., \& Homberg, U. (2014). Organization and functional roles of the central complex in the insect brain. Annual Review of Entomology, 59, 165-184.

Plath, J. A., \& Barron, A. B. (2015). Current progress in understanding the functions of the insect central complex. Current Opinion in Insect Science, 12, 11-18.

Saidel, W. M. (2009). Evolution of the optic tectum in anamniotes. In M. D. Binder, N. Hirokawa, \& U. Windhorst (Eds.), Encyclopedia of neurosciences (pp. 1380-1387). Berlin: Springer-Verlag.

Strausfeld, N. J., \& Hirth, J. (2013). Deep homology of arthropod central complex and vertebrate basal ganglia. Science, 340, 157-161.

Wilczynski, W., \& Northcutt, R. G. (1983). Connections of the bullfrog striatum: Afferent organization. Journal of Comparative Neurology, 214(3), 321-332.

Wulliman, M. F., \& Vernier, P. (2009). Evolution of the telencephalon in anamniotes. In M. D. Binder, N. Hirokawa, \& U. Windhorst (Eds.), Encyclopedia of neurosciences (pp. 14231431). Berlin: Springer-Verlag. 